\title{
Long noncoding RNAs expression profile and functional networks in rheumatoid arthritis
}

\author{
Donghua Xu ${ }^{1,2, *}$, Ye Jiang ${ }^{3, *}$, Lu Yang ${ }^{3, *}$, Xixing Hou ${ }^{1}$, Jihong Wang $^{1}$, Weijun Gu ${ }^{1}$, \\ Xiaodong Wang ${ }^{1}$, Lanyu Liu ${ }^{4}$, Juan Zhang ${ }^{5}$ and Hongying Lu ${ }^{6}$ \\ ${ }^{1}$ Department of Rheumatology and Immunology, The Affiliated Hospital of Weifang Medical University, Weifang 261000, \\ China \\ ${ }^{2}$ Clinical Medicine College of Weifang Medical University, Weifang 261000, China \\ ${ }^{3}$ Department of Laboratory Medicine, The First Affiliated Hospital of Nanjing Medical University, Nanjing 210029, China \\ ${ }^{4}$ Department of Gynecology and Obstetrics, Weifang Hospital of Maternal and Child Health, Weifang 261000, Shandong \\ Province, China \\ ${ }^{5}$ Department of Rehabilitation, Affiliated Huai'an Hospital of Xuzhou Medical College and Second People's Hospital \\ of Huai'an, Huai'an 223001, China \\ ${ }^{6}$ Functional Laboratory, Clinical Medicine College of Weifang Medical University, Weifang 261000, China \\ *These authors contributed equally to this work
}

Correspondence to: Hongying Lu, email: luhy1030@163.com Juan Zhang, email: 1299878898@qq.com

Keywords: long noncoding RNAs, rheumatoid arthritis, toll like receptor

Received: June 10, $2017 \quad$ Accepted: July 25, $2017 \quad$ Published: August 08, 2017

Copyright: $\mathrm{Xu}$ et al. This is an open-access article distributed under the terms of the Creative Commons Attribution License 3.0 (CC BY 3.0), which permits unrestricted use, distribution, and reproduction in any medium, provided the original author and source are credited.

\section{ABSTRACT}

The modifying effects of long noncoding RNAs (IncRNAs) in rheumatoid arthritis (RA) recently have drawn much attention; however, the underlying mechanisms remain largely unknown. Herein, we aim to investigate the expression profile of IncRNAs in RA and identify promising targets for RA diagnosis and treatment. Microarray screening and real-time PCR of IncRNAs were performed by use of serum samples from 3 RA patients and 3 healthy controls. Significantly differentially expressed IncRNAs were verified in serum samples from 43 RA patients and 40 healthy controls by real-time PCR. We found that there were 73 up-regulated and 61 down-regulated IncRNAs as well as 128 up-regulated and 37 down-regulated mRNAs in serum samples of RA patients. Validation in RA clinical samples indicated 5 of these IncRNAs were significantly up-regulated including RNA143598, RNA143596, HIX0032090, IGHCgamma1, and XLOC_002730. Significant association was observed between these IncRNAs and the disease course, erythrocyte sedimentation rate (ESR), rheumatoid factor (RF) as well as anti-cyclic citrullinated peptide (anti-CCP) antibody. Additionally, 55 of the differentially expressed mRNAs were associated with 41 IncRNAs and were involved in signaling pathways of toll like receptors (TLRs), nuclear factor-kappa B (NF-KB), and cytokine, especially the IRF3/IRF7 mediated signaling transduction. Our study firstly shows the specific profile of IncRNAs in the serum of RA patients and potential signaling pathways involved in RA pathogenesis, which may provide novel targets for the diagnosis and treatment of patients with RA.

\section{INTRODUCTION}

Rheumatoid arthritis (RA) is a common autoimmune inflammatory disease, whereas the pathogenesis of it is not fully elucidated. RA is characteristic of systemic and chronic inflammation, abnormal immune response, and irreversible joint destruction $[1,2]$. Some extra-articular manifestations, such as cardiovascular diseases, interstitial lung disease and polyangitis, often lead to poor prognosis of patients with RA [3-5]. Accumulated evidence has 
suggested that aberrant activation of innate and adaptive immune response plays vital roles in the development and progression of RA. Imbalance of cytokines is involved in RA. Many studies have implicated that a variety of cytokines including TNF-a, IL-1, IL-6, IL-17, IL-18, IL-29, IL-33 are involved in the pathogenesis of RA $[6,7]$. Thus, biologics targeting those inflammatory cytokines have been extensively investigated and applied to the treatment of RA, such as TNF-a and IL-6. As biotechnology and bioinformatics grow, research in the expression and regulation of human genes in RA has drawn wide attention. During the past few years, increasing evidence has suggested that the abnormal expression and dysregulation of long noncoding RNAs (lncRNAs) may participate in the pathogenesis of autoimmune diseases including RA [8,9] .

LncRNAs are RNA transcripts with more than 200 nucleotides in length. They are a new class of regulatory RNAs that are not translated into proteins. LncRNAs are transcription products of RNA polymerase II, which are widely expressed in normal tissues of human body and may be aberrantly and specifically expressed in certain pathological tissues. A number of studies have implicated that lncRNAs can be transported and released into the periphery by circulating exosomes, mediating interacts between cells and their microenvironment $[10,11]$. During the last decade, lncRNAs has been strongly suggested to be involved in the development of several kinds of diseases, such as cancer, cardiovascular diseases, and rheumatoid diseases [12-14]. Emerging evidence has revealed that lncRNAs are involved in the regulation of certain biological processes, including chromatin remodeling, gene transcription, and protein transport [15]. It has been well established that lncRNAs regulate the differentiation and activation of T cells, B cells, macrophages, and NK cells, and thus affect autoimmunity and immune-related diseases, such as RA, systemic lupus erythematosus (SLE), primary Sjögren's syndrome (pSS), psoriasis, polymyositis/dermatomyositis (PM/DM) and Crohn's disease (CD) [9, 16, 17]. Therefore, elucidating the role of IncRNAs in RA can help to understand the pathogenesis of RA and provide novel promising targets for the diagnosis, treatment and prognosis estimation of RA.

The aim of this study is to investigate the underlying effects of lncRNAs in RA by microarray screening and bioinformatics analysis. The findings will provide new insights into the pathogenesis of RA and help to identify prospective targets for RA.

\section{RESULTS}

\section{Aberrantly expressed IncRNAs and mRNAs in $\mathbf{R A}$}

The hierarchical clustering analysis, scatter plot and volcano plot all showed that some lncRNAs and mRNAs were differentially expressed in the serum of RA patients compared with healthy controls (Figure 1A-1C and Figure 2A-2C). A total of 73 up-regulated and 61 downregulated lncRNAs as well as 128 up-regulated and 37 down-regulated mRNAs were identified in the serum of patients with RA after the microarray screening. Table 1 and Table 2 presented the top 30 aberrantly expressed lncRNAs and mRNAs in RA, respectively.

\section{LncRNA expression profile in the serum of RA}

Most of the aberrantly expressed lncRNAs were validated in the serum samples from 43 RA patients and 40 healthy controls by real-time PCR. 5 of these lncRNAs were found to be significantly up-regulated in the serum of RA patients when validating by real-time PCR, including RNA143598, RNA143596, HIX0032090, IGHCgamma1, and XLOC_002730 (Figure 3).

\section{Association between differentially expressed IncRNAs and clinical characteristics of RA}

Significant association was observed between these up-regulated lncRNAs and the disease course, erythrocyte sedimentation rate (ESR), rheumatoid factor (RF), anticyclic citrullinated peptide antibody (anti-CCP Ab) of RA (Figure 4).

\section{Functional prediction of aberrantly expressed mRNAs}

Figure 5A showed the top 30 significant enriched pathway terms with regard to the aberrantly expressed mRNAs in RA, primarily including toll like receptors (TLRs), nuclear factor-kappa B (NF- $\kappa \mathrm{B})$, and cytokine signaling pathways. Besides, the interferon regulatory factor (IRF3/IRF7) mediated signaling transduction was dominant in those pathways. The top 30 significant enriched GO terms were presented in Figure 5B, which were mainly involved in the biological processes, cellular components, and molecular functions of aberrantly expressed mRNAs in RA.

\section{Association between IncRNAs and mRNAs}

Computational analysis revealed that 55 mRNAs were associated with 41 differentially expressed lncRNAs (Figure 6). These significantly associated lncRNAsmRNAs pairs were primarily involved in signaling pathways of TLRs, NF- $\kappa \mathrm{B}$, and cytokine, which might contribute to the pathogenesis of RA and influence the prognosis of RA patients.

\section{DISCUSSION}

LncRNAs, as a class of noncoding RNAs, play critical roles in the regulation of autoimmunity and 
Table 1: Top 30 aberrantly expressed IncRNAs in RA patients compared with healthy controls

\begin{tabular}{|c|c|c|}
\hline LncRNA & $P$ value & Fold changes \\
\hline \multicolumn{3}{|l|}{ Up-regulated } \\
\hline RNA147405|p0509_imsncRNA82 & 0.034815 & 7.772733 \\
\hline ENST00000561134.1 & 0.037058 & 6.968304 \\
\hline НIT000064601 & 0.015806 & 6.695212 \\
\hline p44304_v4 & 0.038641 & 5.674733 \\
\hline RNA143598 & 0.023631 & 5.673976 \\
\hline XR_243720.2 & 0.034541 & 5.649367 \\
\hline XR_429995.1 & 0.034812 & 5.621383 \\
\hline ENST00000579527.1 & 0.01753 & 5.498751 \\
\hline p28385 & 0.040602 & 5.334821 \\
\hline TCONS_00007197 & 0.019452 & 5.043555 \\
\hline RNA143577 & 0.021894 & 5.00845 \\
\hline RNA143578 & 0.030799 & 4.922104 \\
\hline RNA143580 & 0.025897 & 4.919801 \\
\hline RNA147303|p0407_imsncRNA822 & 0.047544 & 4.911092 \\
\hline RNA143589 & 0.023075 & 4.780086 \\
\hline RNA143581 & 0.023414 & 4.638907 \\
\hline HIX0032090 & 0.013736 & 4.616553 \\
\hline RNA143595 & 0.022496 & 4.565024 \\
\hline RNA143579 & 0.027482 & 4.386317 \\
\hline LIT3528 & 0.00859 & 4.378436 \\
\hline RNA143540|tRNA_457_68 & 0.031161 & 4.270831 \\
\hline RNA143596 & 0.040198 & 4.074681 \\
\hline ENST00000548571.1 & 0.043473 & 3.740273 \\
\hline XR_108761.3 & 0.016348 & 3.731026 \\
\hline LIT3526 & 0.037608 & 3.678755 \\
\hline ENST00000553426.1 & 0.016396 & 3.518957 \\
\hline HIT000092395_03 & 0.039359 & 3.492784 \\
\hline ENST00000441075.1 & 0.023534 & 3.432525 \\
\hline IGHCgamma1 & 0.025053 & 3.284312 \\
\hline XLOC_002730 & 0.030258 & 3.222785 \\
\hline \multicolumn{3}{|l|}{ Down-regulated } \\
\hline p26558 & 0.034042 & 6.986983 \\
\hline ENST00000541797.1 & 0.005979 & 4.39359 \\
\hline HIT000296997 & 0.016428 & 4.269773 \\
\hline RNA33664|snoRNA_scaRNA_260_79 & 0.007309 & 4.121303 \\
\hline TCONS_00022112 & 0.046352 & 3.983995 \\
\hline ENST00000495580.1 & 0.001753 & 3.581599 \\
\hline ENST00000439406.1 & 0.008247 & 3.336626 \\
\hline RNA95721|RNS_803_121 & 0.028929 & 3.264407 \\
\hline XR_242051.1 & 0.012273 & 3.085937 \\
\hline TCONS_00027142 & 0.041577 & 3.062286 \\
\hline ENST00000606966.1 & 0.004208 & 2.963269 \\
\hline
\end{tabular}




\begin{tabular}{lcc} 
ENST00000525331.1 & 0.047289 & 2.935378 \\
ENST00000606879.1 & 0.018672 & 2.90775 \\
ENST00000596887.1 & 0.033564 & 2.898185 \\
ENST00000439633.1 & 0.005507 & 2.886541 \\
TCONS_00009147 & 0.026974 & 2.801348 \\
ENST00000422944.1 & 0.040728 & 2.796731 \\
ENST00000550263.1 & 0.036059 & 2.751807 \\
TCONS_00021834 & 0.037043 & 2.744497 \\
ENST00000586610.1 & 0.032215 & 2.668683 \\
TCONS_00027516 & 0.032387 & 2.652956 \\
ENST00000436429.1 & 0.025568 & 2.635222 \\
TCONS_00021014 & 0.033183 & 2.536658 \\
ENST00000525429.1 & 0.041101 & 2.507953 \\
ENST00000450990.1 & 0.01297 & 2.496252 \\
ENST00000563230.1 & 0.017314 & 2.493657 \\
TCONS_00028421 & 0.00096 & 2.467011 \\
XR_109933.1 & 0.012023 & 2.44694 \\
ENST00000604448.1 & 0.024131 & 2.442996 \\
nc-HOXC11-108 & 0.039676 & 2.400215 \\
\hline
\end{tabular}

Table 2: Top 30 differentially expressed mRNAs in RA patients compared with healthy controls

\begin{tabular}{lcc}
\hline mRNA & P value & Fold changes \\
\hline Up-regulated & & \\
CST5 & 0.011015 & 11.7354 \\
MYL5 & 0.016609 & 9.437377 \\
MTRNR2L2 & 0.017951 & 9.170133 \\
ABLIM3 & 0.035346 & 8.106463 \\
ATP5A1 & 0.028899 & 8.078051 \\
CDK2AP1 & 0.028824 & 8.064926 \\
TRAPPC1 & 0.030183 & 7.857362 \\
HLA-A & 0.002232 & 7.815082 \\
MAP1LC3B & 0.036902 & 7.535449 \\
MNDA & 0.039922 & 7.465274 \\
CD9 & 0.02348 & 7.367939 \\
GABARAPL2 & 0.028315 & 7.146566 \\
ATP6V0C & 0.036303 & 7.128572 \\
FCGR2A & 0.040717 & 7.009223 \\
OSBPL9 & 0.038812 & 6.904492 \\
RAB11B & 0.042927 & 6.857076 \\
MTRNR2L9 & 0.045445 & 6.854809 \\
ND4L & 0.016292 & 6.695147 \\
DUSP6 & 0.042244 & 6.100676 \\
TLN1 & 0.031514 & 5.967558 \\
GNG10 & 0.034438 & 5.903362
\end{tabular}


MLH3

CDC14B

LOC100130865

PTPN22

A_21_P0013791

FURIN

TCP11L2

USP17L25

ND4

Down-regulated

ACACA

RAB6C-AS1

DNAH2

GGCT

TRIP10

CSF1R

TMEM229B

FILIP1L

A_33_P3244828

lnc-GRIK1-AS2-1

BCL6

INPP5F

NUTM2B

CYP2R1

NELL1

A_33_P3398005

HOXD10

A_33_P3333364

ZNF358

A_33_P3290301

POU4F3

lnc-CIB4-1

$\mathrm{TMCO} 2$

SSX3

A_33_P3287058

lnc-RASA1-5

ADCYAP1R1

SP140

lnc-TEX261-2

MGAT4D
0.016582

0.030726

0.04633

0.018824

0.029625

0.048091

0.042912

0.000509

0.044486

0.020405

0.028936

0.022414

0.037562

0.006481

0.047671

0.018878

0.008344

0.034094

0.022228

0.030188

0.046803

0.010629

0.019688

0.003652

0.00733

0.019771

0.01795

0.017607

0.017789

0.04991

0.017052

0.04851

0.017698

0.006785

0.003285

0.043844

0.041264

0.034831

0.028391
5.829806

5.664988

5.565929

5.555936

5.535323

5.514076

5.49967

5.468273

5.341476

4.04911

3.383973

3.373326

3.187807

3.142754

3.142102

2.677905

2.559852

2.470736

2.376733

2.364881

2.35886

2.329807

2.279476

2.272997

2.235301

2.223335

2.191913

2.185415

2.182656

2.171979

2.145348

2.144128

2.13033

2.109284

2.092431

2.063525

2.060501

2.046709

2.041796 maintenance of homeostasis [18, 19]. Multiple lines of evidence have suggested that the dysregulation of lncRNAs is involved in a variety of human diseases, including metabolic disease, cancer and rheumatoid disease [19-21]. A spectrum of mechanisms has been elucidated, such as the control of mRNA decay, recruitment of epigenetic modifier proteins, and regulation of microRNAs expression [21-23]; however, mechanisms 
of lncRNAs-mediated regulation in RA remain not fully understood. Understanding of lncRNAs-mediated regulation is essential for investigating prospective and novel targets for the diagnosis, treatment and prognosis estimation of RA.

RA is one of the most frequent rheumatoid diseases worldwide with kinds of strong autoimmune components, such as autoantigens of citrullinated proteins [2, 3]. To the best of our knowledge, inflammation is closely related to the occurrence of RA, disease activity and prognosis. Long sustained and chronic autoimmune inflammation in the synovium can lead to destruction of joints and deformity. LncRNAs are involved in the expression of inflammatory related genes, epigenetics, inflammatory signal transduction and other biological processes by acting as guidance molecules, signal molecules, decoy molecules, and cytoskeleton molecules [24]. Therefore, it can be concluded that lncRNAs may confer modifying effects on the development and progression of RA. Recent progress has suggested that the role of lncRNAs in RA could be far more prevalent than previously appreciated. LncRNA ANRIL is the first documented lncRNA involved in the pathogenesis of RA [25]. Additionally, IncRNA ANRIL can influence inflammation primarily by modulating $\mathrm{NF}-\kappa \mathrm{B}$ and its downstream signaling pathway, and thus participates in biological processes of glucose metabolism and inflammatory response and ultimately causes coronary heart disease, vasculitis and other inflammation-related diseases [25, 26]. Aterido A et al. have found that IncRNA FAM66C was abnormally expressed in CD4+ $\mathrm{T}$ lymphocytes and could affect CD4+ $\mathrm{T}$ cells-mediated immune response in RA [27]. Increased expression of IncRNA Hotair can induce the migration of more macrophages to inflammatory sites and

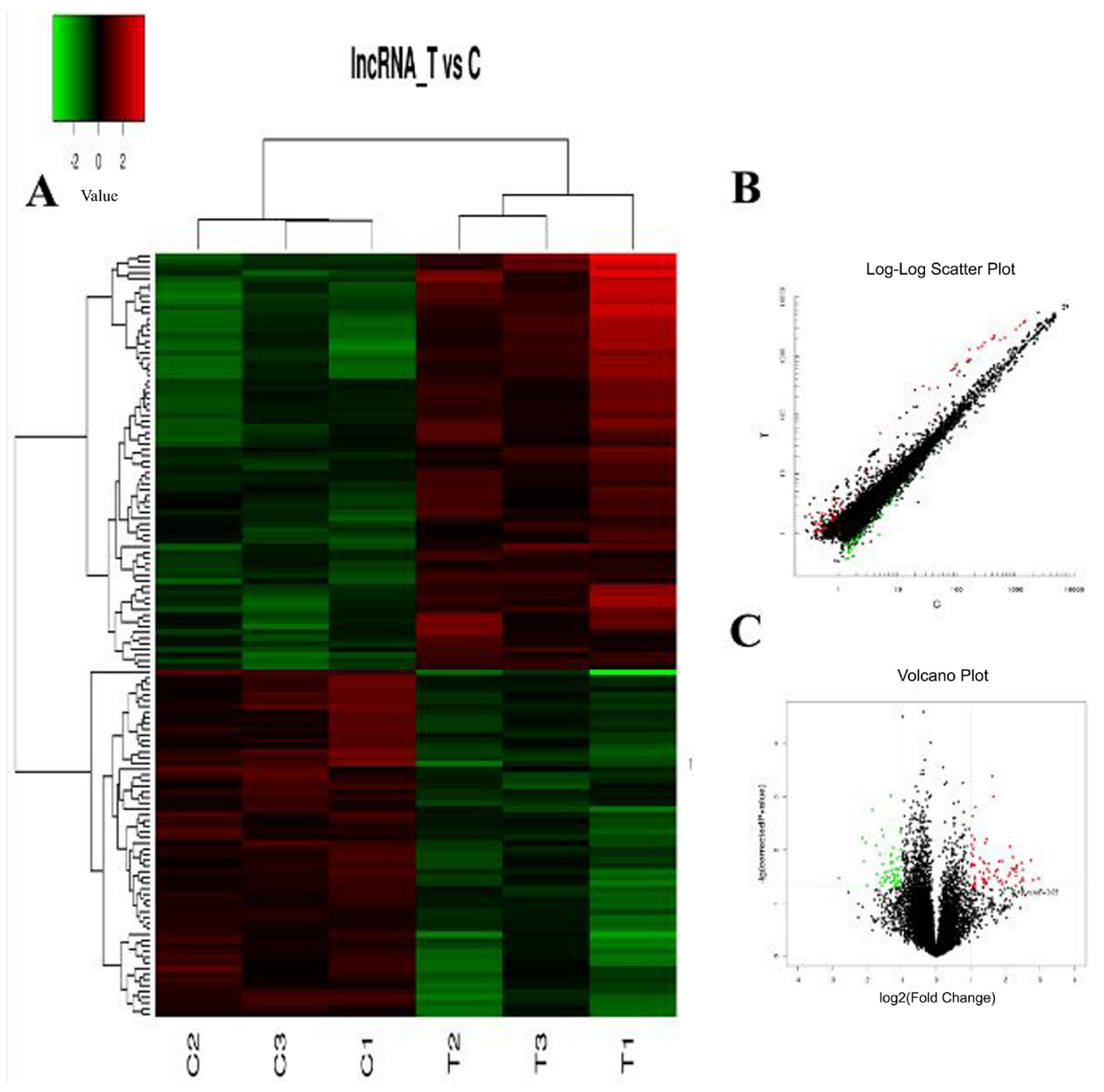

Figure 1: The expression profile of IncRNAs in RA patients compared with healthy controls. (A) Hierarchical clustering analysis presenting differently expressed lncRNAs. Colors of red and green represent up- and down-regulated genes with changes larger than twofold, respectively. (B) Scatter plot showing differently expressed lncRNAs. Red and green plots represent up- and down-regulated genes with changes larger than twofold, respectively. (C) Volcano plot showing differently expressed lncRNAs. Red and green plots represent up- and down-regulated genes with changes larger than twofold, respectively. 
the amplification of local inflammatory response, which ultimately promotes the progression of RA [28]. Taken together, lncRNAs-mediated inflammatory response and immune regulation play crucial roles in the development and progression of RA. Nonetheless, little is known about the molecular mechanism in RA pathogenesis regarding lncRNAs-mediated modulation, which warrants further investigation in more future studies.

In the present study, we tried to find the differentially expressed lncRNAs and mRNAs profiles in the serum of RA patients by microarray screening. 5 significantly differentially expressed lncRNAs were identified in serum samples from patients with RA, including RNA143598, RNA143596, HIX0032090, IGHCgamma1, and XLOC_002730. Besides, significantly positive association was observed between these lncRNAs and the disease course, ESR, RF and anti-CCP antibody of patients with RA. The correlation analysis showed that RNA143598 and HIX0032090 were significantly associated with the disease course and ESR level in patients with RA. In addition, increased levels of RF were observed in RA patients with XLOC_002730 and HIX0032090 up-regulated in the serum among RA patients. Moreover, the expression of
RNA143596, RNA143598 and HIX0032090 was also positively related to the level of anti-CCP antibody in the serum. It has been well established that elevated level of $\mathrm{RF}$ is highly associated with synovitis, vasculitis and extra articular symptoms, while increased level of anti-CCP antibody is closely related to bone erosion and destruction [29]. Accordingly, all these findings have suggested important values of lncRNAs in the diagnosis and prognosis adjustment of RA. The differentially expressed lncRNAs are associated with inflammatory response and autoimmunity, and thus influence RA occurrence, progression and prognosis.

As shown in the pathway enrichment analysis, TLRs/NF- $\kappa$ B signal pathways are highly enriched in RA associated with the aberrantly expressed mRNAs. Thus, TLRs/NF- $\kappa \mathrm{B}$ mediated inflammation may contribute to the development of RA. Currently published studies have suggested a crucial role of TLRs/NF- $\kappa \mathrm{B}$ signaling transduction in the development of some autoimmune diseases including RA, Sjogren's syndrome and systemic sclerosis [30-32], supporting multiple promising therapeutic targets for these diseases. Similarly, we have previously found that LPS/TLR4/NF- $\kappa B$ signaling

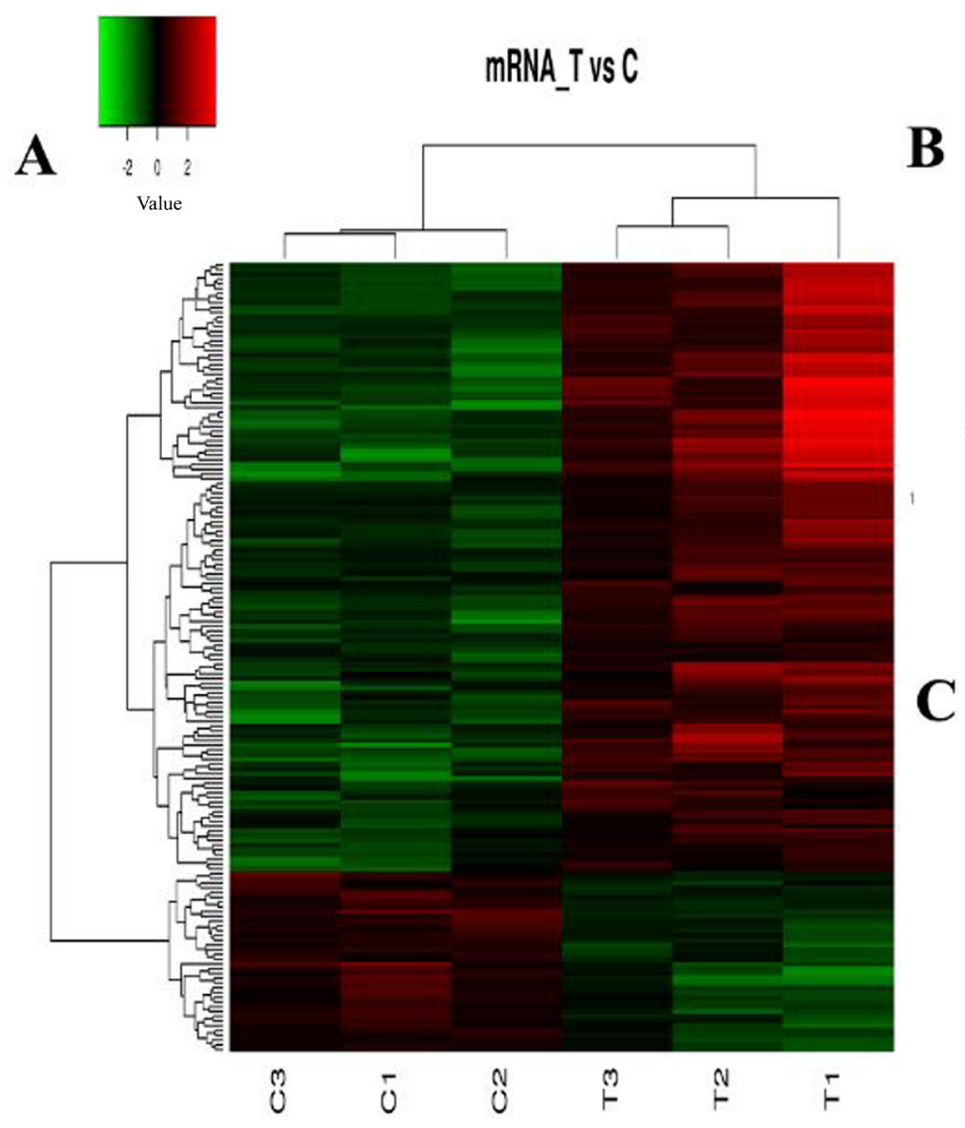

B
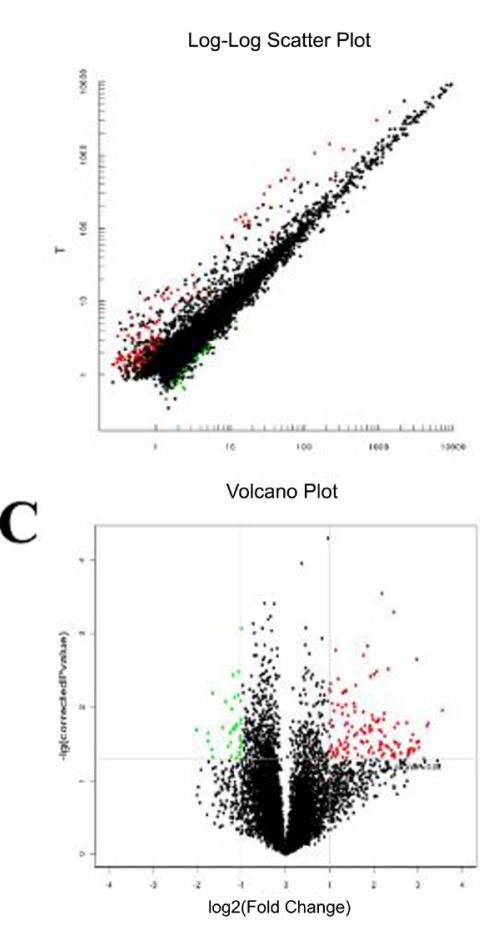

Figure 2: The expression profile of mRNAs in RA patients compared with healthy controls. (A) Hierarchical clustering analysis presenting differently expressed mRNAs. Colors of red and green represent up- and down-regulated genes with changes larger than twofold, respectively. (B) Scatter plot showing differently expressed mRNAs. Red and green plots represent up- and down-regulated genes with changes larger than twofold, respectively. (C) Volcano plot showing differently expressed mRNAs. Red and green plots represent upand down-regulated genes with changes larger than twofold, respectively. 
pathway contributed to the pathogenesis of RA [7]. Taken together, LPS/TLR4-mediated inflammation is involved in RA dependent on NF- $\kappa \mathrm{B}$ signaling activation. Nonetheless, except for TLR4, other common types of TLRs, such as TLR3, TLR7, and TLR9, may also contribute to the RA pathogenesis, which is needed to be further investigated in more future studies, particularly regarding the underlying molecular mechanisms of lncRNAs involved in RA pathogenesis.

In this study, the cytokine signaling pathway has been significantly enriched by the bioinformatics analysis in the present study, especially the IRF3/IRF7 mediated signaling transduction. IRF-3 and IRF-7 are the vital transcriptional factors for the generation of IL-28A and IL-28B, whereas IRF3 and NF- $\mathrm{KB}$ are critical transcript factors for the production of the IL-29 [33]. IL-29, an important molecule of type III interferon family, has been demonstrated to be associated with enhanced inflammation in the development of RA in our previous study [7]. Type III interferon mainly consists of IL-29, IL-28A and IL-28B. In addition, several published studies have implicated that IL-29 could affect the inflammatory response involved in the pathogenesis of certain diseases by activation of IRFs signaling pathways $[33,34]$. Moreover, the study by $\mathrm{Xu}$ et al. has revealed that IL-29 might enhance TLRs-mediated production of inflammatory cytokines in synovial fibroblasts [30], suggesting a crucial role of IL-29/TLRs signaling pathways in RA pathogenesis. However, the potential effects of type III interferon family and IRFs related signaling transduction in RA remain largely unknown. We hypothesize that IL-29/IRFs signaling pathways might participate in the development and progression of RA. However, we fail to elucidate the underlying effects of IncRNAs/IL-29/IRFs in RA by use of cell and/or animal models in this study. More future studies are warranted to demonstrate the precise regulatory mechanisms of lncRNAs/IL-29/IRFs signal involved in RA.

In summary, this study, for the first time, shows specific profiles of lncRNAs and mRNA in the serum of RA patients and potential lncRNA-mRNA networks involved in RA. There are a total of 73 up-regulated and 61 down-regulated lncRNAs as well as 128 up-regulated and 37 down-regulated mRNAs in RA. LncRNAs of RNA143598, RNA143596, HIX0032090, IGHCgamma1, and XLOC_002730 are significantly up-regulated in the serum of RA patients. The differentially expressed lncRNAs are closely associated with the inflammatory response and autoimmunity. Findings in this study will support novel promising targets for RA.
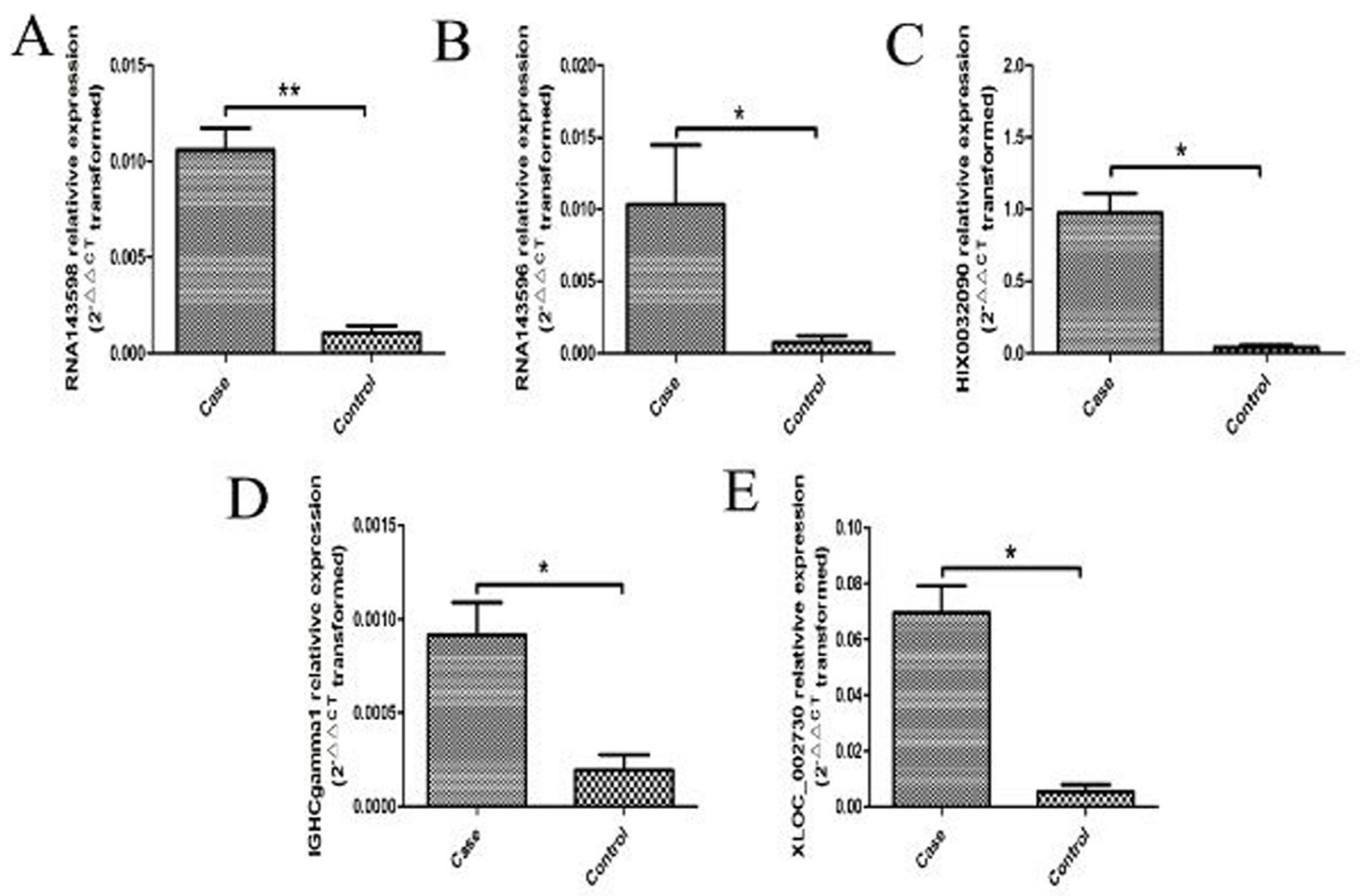

Figure 3: 5 aberrantly expressed IncRNAs validated by real-time PCR in the serum from 43 RA patients and 40 healthy controls. (A) RNA143598 was significantly increased in RA compared with healthy controls $\left({ }^{* *} P<0.01\right)$. (B) RNA143596 was significantly increased in RA compared with healthy controls $\left({ }^{*} P<0.05\right)$. (C) HIX0032090 was significantly increased in RA compared with healthy controls $\left({ }^{*} P<0.05\right)$. (D) IGHCgammal was significantly increased in RA compared with healthy controls $\left({ }^{*} P<0.05\right)$. (E) XLOC_002730 was significantly increased in RA compared with healthy controls $\left({ }^{*} P<0.05\right)$. 

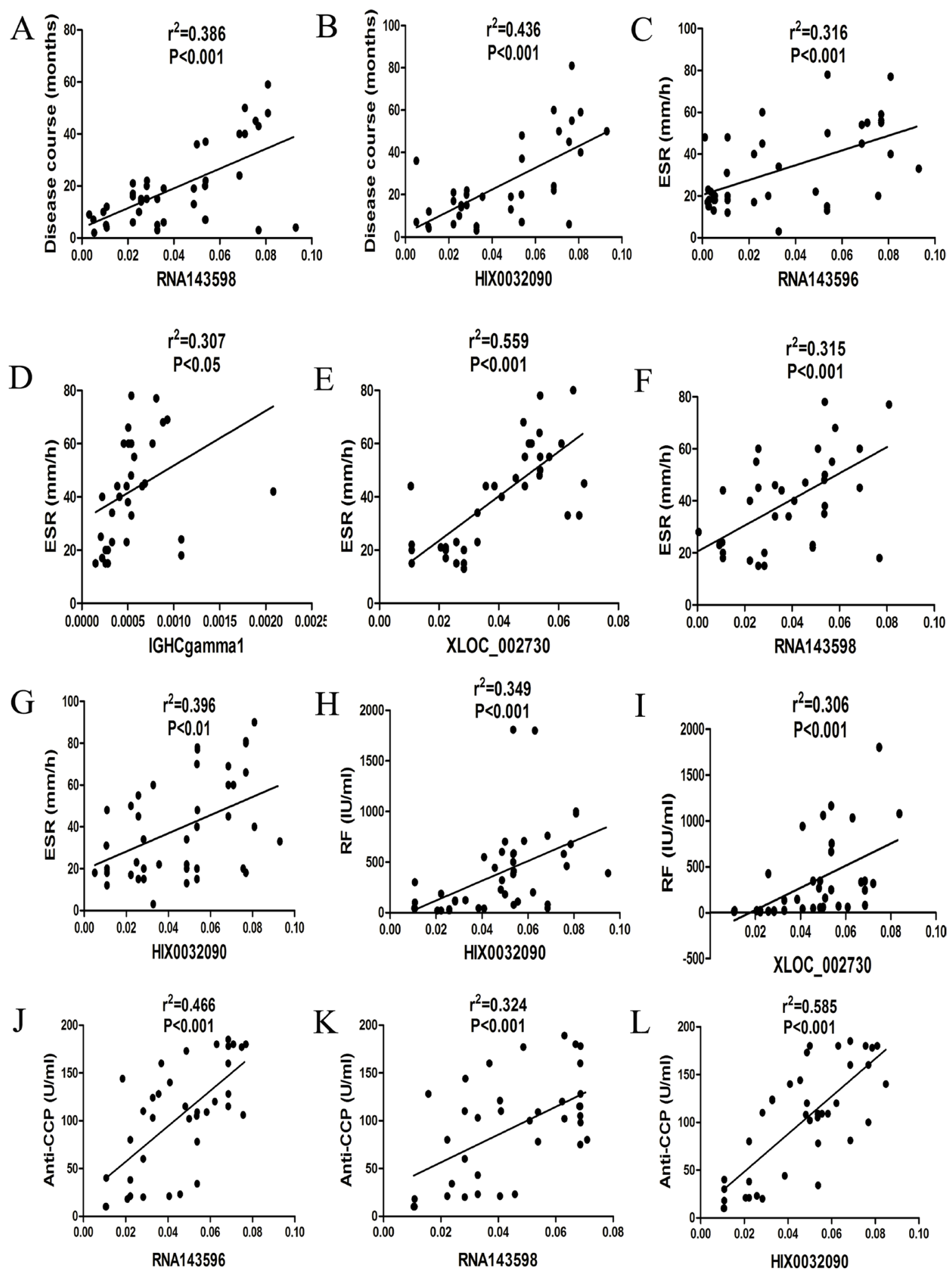

Figure 4: Correlations between aberrantly expressed IncRNAs and clinical characteristics of RA. (A) RNA143598 was significantly related to the disease course of RA. (B) HIX0032090 was significantly related to the disease course of RA. (C) RNA143596 was significantly related to ESR in RA. (D) IGHCgamma1 was significantly related to ESR in RA. (E) XLOC_002730 was significantly related to ESR in RA. (F) RNA143598 was significantly related to ESR in RA. (G) HIX0032090 was significantly related to ESR in RA. (H) HIX0032090 was significantly related to RF in RA. (I) XLOC_002730 was significantly related to RF in RA. (J) RNA143596 was significantly related to anti-CCP Ab in RA. K RNA143598 was significantly related to anti-CCP Ab in RA. (L) HIX0032090 was significantly related to anti-CCP Ab in RA. 
Constitutive Signaling by Ligand-Responsive EGFR Cancer Variants | REACT_115852

Activation of IRF3/RF7 mediated by TBK1/KK epsilon | REACT_25148

Oxytocin signaling pathway | hsa04921

Parkinson's disease | hs 05012

TRAF6 mediated IRF7 activation in TLR7/8 or 9 signaling | REACT_25120 TRAF6 mediated induction of TAK1 complex | REACT 2535 p75NTR signals via NF-KB | REACT_13537

TGF-beta receptor signaling in EMT (epithelial to mesenchymal transition) | REACT_120726 Tight junction | hsa04530 Regulation of innate immune responses to cytosolic DNA | REACT_163977 NRIF signals cell death from the nucleus | REACT_ 13643 Constitutive Signaling by NOTCH1 HD Domain Mutants | REACT_160089 IRAK1 recruits IKK complex | REACT 2491 Glycogen synthesis | REACT_16920 Association of licensing factors with the pre-replicative complex | REACT 118 IRAK1 recruits IKK complex upon TLR7/8 or 9 stimulation | REACT_25354 Vascular smooth muscle contraction | hsa04270 Leukocyte transendothelial migration | hsa04670 NF-kB is activated and signals survival | REACT_ 1369 p75NTR recruits signalling complexes | REACT_ 13415 Assembly Of The HIV Virion | REACT_6818 Spry regulation of FGF signaling $\mid$ REACT_ 111080 Downregulation of ERBB2:ERBB3 signaling | REACT_115662 Synthesis And Processing of GAG, GAGPOL Polyproteins | REACT_115860 Membrane binding and targetting of GAG proteins | REACT_11589 IRAK2 mediated activation of TAK1 complex | REACT_2538 Regulation of the Fanconi anemia pathway | REACT_18265 IRAK2 mediated activation of TAK1 complex upon TLR7/8 or 9 stimulation | REACT_25018
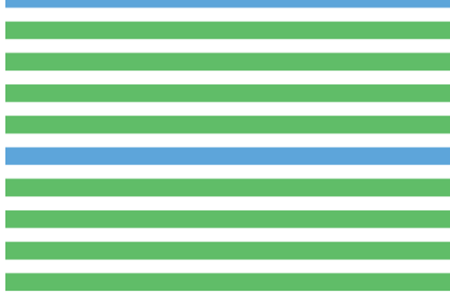
然

B

\begin{tabular}{|c|c|c|c|}
\hline : & $\stackrel{\circ}{\circ}$ & -log(Corrected p-Value) & $\stackrel{n}{\square}$ \\
\hline & & nt Enriched GO Ter & 30) \\
\hline
\end{tabular}

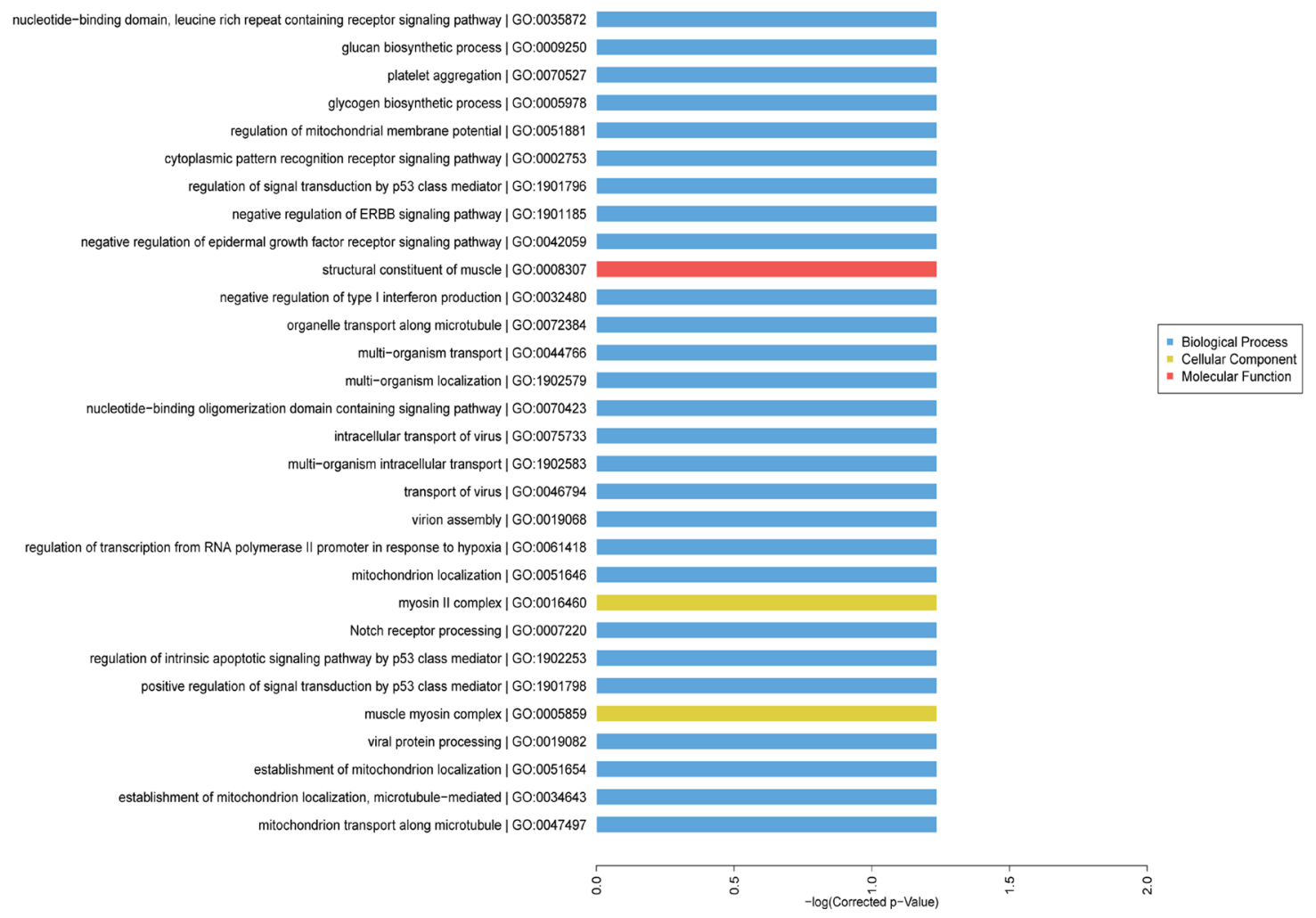

Figure 5: GO and pathway enriched analyses for differently expressed mRNAs. (A) Significantly enriched pathway terms of differently expressed mRNAs.(B) Significantly enriched GO terms of differently expressed mRNAs. 


\section{MATERIALS AND METHODS}

\section{Study subjects and sample preparation}

43 RA patients and 40 healthy controls were recruited from the affiliated hospital of Weifang Medical University between September, 2015 and March, 2016. Controls were from the same hospital for health examination. All fresh blood samples were separately collected and sequentially centrifuged at $2000 \mathrm{rpm}$ for $10 \mathrm{~min}$. $1 \mathrm{ml}$ cell-free serum from the supernatant was sucked out and stored at $-80^{\circ} \mathrm{C}$ for further detection. Characteristics of all patients and controls were summarized in Table 3.

\section{RNA isolation and real-time PCR}

Total RNAs were isolated from serum samples by use of Plasma/Serum RNA Purification Mini Kit (Norgen Biotek Corp., Thorold, Canada) according to the manufacturers' protocols. Purified total RNAs were quantified using a NanoDrop 1000 (Thermo Fisher Scientific, Waltham, MA, USA). Then, cDNAs were synthesized from $0.5 \mu \mathrm{g}$ RNAs for further assay based on the instructions of PrimeScript ${ }^{\mathrm{TM}} \mathrm{RT}$ reagent Kit (Takara, Tianjin, China). Real-time PCR was carried out in triplicate assay in accordance with the specifications of SYBR Green Mastermix kit (Takara, Tianjin, China). A total of $5 \mathrm{ng}$ cDNA template was used for real-time PCR assay. Random primers were used in experiments. The expression of each lncRNA was represented as fold changes using the $2^{-\triangle \Delta C T}$ method and normalized to housekeeping gene GAPDH. Primer sequences used in validation of lncRNAs in this paper were listed in Table 4.

\section{Microarray screening}

Capitalbio Agilent LncRNA + mRNA Human Gene Expression Microarray V4.0 was used to screen differentially expressed lncRNAs and mRNAs in the serum of RA patients (Capitalbio Corp., Beijing, China). In this study, serum samples from 3 patients and 3 controls were

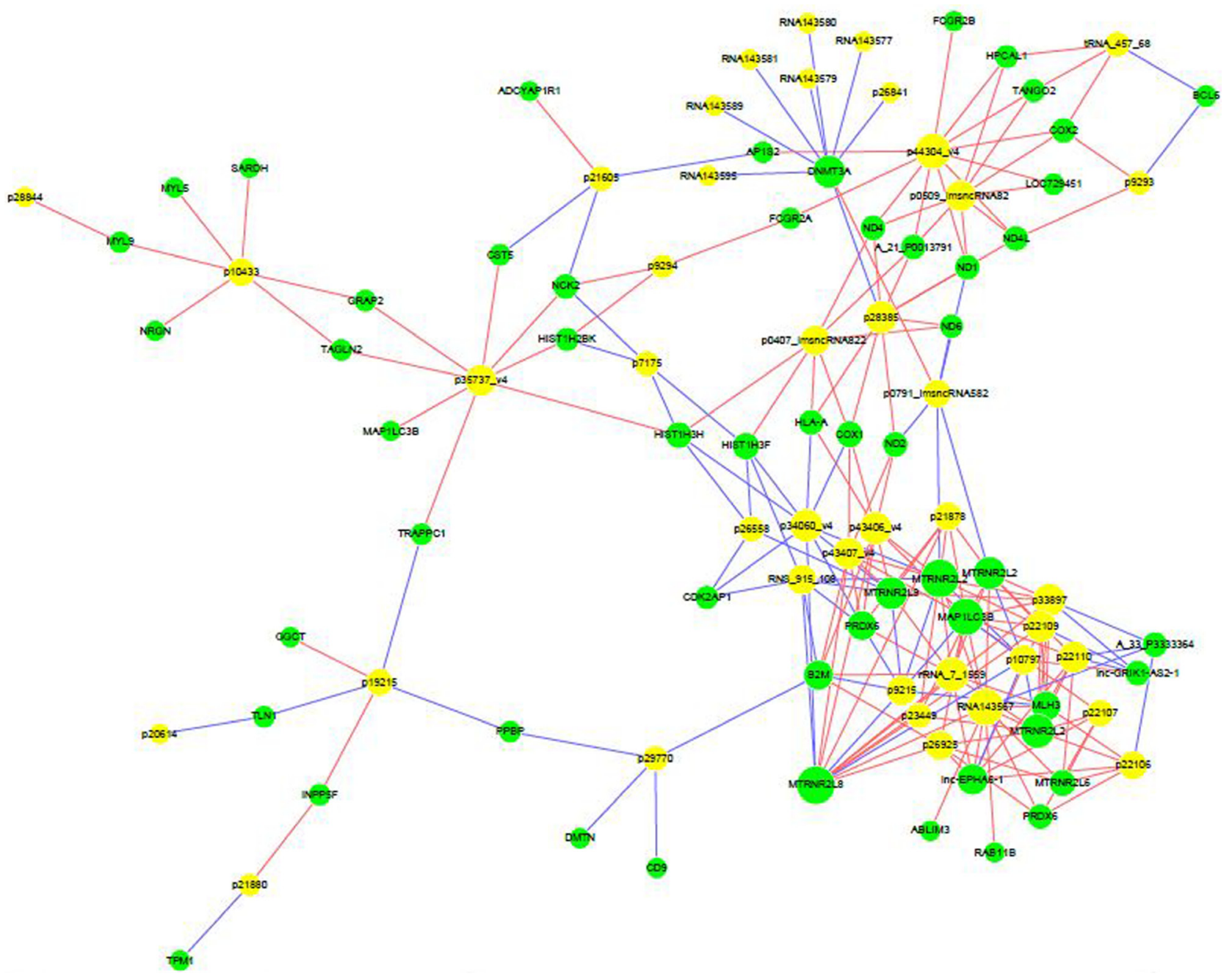

Figure 6: Correlation between IncRNAs and mRNAs. LncRNA-mRNA network graph showed that 55 differentially expressed mRNAs were associated with 41 lncRNAs. 
Table 3: Characteristics of RA patients and controls

\begin{tabular}{lcc}
\hline & Patients $(\boldsymbol{n}=\mathbf{4 3})$ & Controls $(\boldsymbol{n}=\mathbf{4 0})$ \\
\hline Age $(\mathrm{mean} \pm \mathrm{SD})$ & $49.2 \pm 19.0$ & $47.8 \pm 17.2$ \\
Sex (Women/Man) & $30 / 13$ & $25 / 15$ \\
Disease course (year) & $2.5 \pm 1.1$ & - \\
Score of disease activity in 28 joints & $3.8 \pm 1.0$ & - \\
ESR $(\mathrm{mm} / \mathrm{h})$ & $57.1 \pm 13.7$ & $11.2 \pm 4.1$ \\
CRP $(\mathrm{mg} / \mathrm{L})$ & $34.1 \pm 5.2$ & $5.1 \pm 1.2$ \\
RF $(\mathrm{IU} / \mathrm{ml})$ & $116.1 \pm 23.4$ & $9.7 \pm 1.0$ \\
Anti-CCP $(\mathrm{U} / \mathrm{ml})$ & $71.5 \pm 19.4$ & $30.9 \pm 14.2$ \\
\hline
\end{tabular}

Table 4: primer sequences used in validation of IncRNAs

\begin{tabular}{ccc}
\hline LncRNA & Up-stream primer sequence (5' to 3') & Down-stream primer sequence (5' to 3') \\
\hline RNA143598 & TTACACAAGCAAGCATCGCC & TATCACTGCTGTCTCCCGTG \\
RNA143596 & CAAAACACTTTGCTCGGCCA & TAATCGTATGGCTGCGGTGG \\
HIX0032090 & ACTGCTCGCCAGAACACTAC & GGTGAGGTTGATCGGGGTTT \\
IGHCgamma 1 & GTGACGGTGTCGTGGAACTC & GTGTTGCTGGGCTTGTGATT \\
XLOC_002730 & TTGCTATGTTATGCCCGCCT & CGGTACCCTAACCGTGCAAA \\
\hline
\end{tabular}

randomly selected for microarray analysis. It was considered to be statistically significant between the two groups when the fold changes for differentially expressed lncRNAs and mRNAs were larger than 2.0 and the $P$ value for $t$-test was less than 0.05. Most of the differentially expressed genes were validated by real-time PCR, especially those lncRNAs which co-expressed with mRNAs.

\section{Bioinformatics analysis}

After microarray screening for lncRNAs and mRNAs in the serum samples from RA patients and controls, the hierarchy clustering, gene ontology (GO) functional enrichment and pathway enrichment analyses were then performed for further estimation by use of KOBAS (KEGG Orthology Based Annotation System) software. In order to predict the potential regulatory effects of differentially expressed lncRNAs on mRNAs, an IncRNA-mRNA co-expression network was conducted by bioinformatics analysis.

\section{Statistical analysis}

Data were shown as mean \pm SEM. We used independent-Samples $\mathrm{T}$ test or One-Way ANOVA for statistical analysis. A two-sided $P<0.05$ was regarded to be statistically significant. Softwares of SPSS (version 16.0) and Graphpad (version 5.0) were applied for statistical analysis.

\section{ACKNOWLEDGMENTS}

None.

\section{CONFLICTS OF INTEREST}

The authors declare no conflicts of interest.

\section{FUNDING}

This work is supported by grants from the National Natural Science Foundation for Young Scholars (Project Number: 81601408), Natural Science Foundation of Shandong Province (Project Number: ZR2016HQ12), and Medical and Health Science and Technology Development Program of Shandong Province (Project Number: 2015WS0052, 2016WS0681 and 2016WS0686), and fund for doctors from Weifang Medical University (02173401).

\section{REFERENCES}

1. Picerno V, Ferro F, Adinolfi A, Valentini E, Tani C, Alunno A. One year in review: the pathogenesis of rheumatoid rthritis. Clin Exp Rheumatol. 2015; 33:551-558.

2. Catrina AI, Joshua V, Klareskog L, Malmstrom V. Mechanisms involved in triggering rheumatoid arthritis. Immunol Rev. 2016; 269:162-174. 
3. Makol A, Matteson EL, Warrington KJ. Rheumatoid vasculitis: an update. Curr Opin Rheumatol. 2015; 27:63-70.

4. O'Dwyer DN, Armstrong ME, Cooke G, Dodd JD, Veale DJ, Donnelly SC. Rheumatoid Arthritis (RA) associated interstitial lung disease (ILD). Eur J Intern Med. 2013; 24:597-603.

5. Zimmerman GA, Weyrich AS. Immunology. Arsonists in rheumatoid arthritis. Science. 2010; 327:528-529.

6. Wang $\mathrm{F}, \mathrm{Xu} \mathrm{L}$, Feng $\mathrm{X}$, Guo D, Tan W, Zhang M. Interleukin-29 modulates proinflammatory cytokine production in synovial inflammation of rheumatoid arthritis. Arthritis Res Ther. 2012; 14:R228.

7. Xu D, Yan S, Wang H, Gu B, Sun K, Yang X, Sun B, Wang X. IL-29 Enhances LPS/TLR4-Mediated Inflammation in Rheumatoid Arthritis. Cell Physiol Biochem. 2015; 37:27-34.

8. Scaria V. Joining the long shots: emerging evidence on the role of long noncoding RNAs in rheumatoid arthritis. Int $\mathrm{J}$ Rheum Dis. 2014; 17:831-833.

9. Wu GC, Pan HF, Leng RX, Wang DG, Li XP, Li XM, Ye DQ. Emerging role of long noncoding RNAs in autoimmune diseases. Autoimmun Rev. 2015; 14:798-805.

10. Liu T, Zhang X, Gao S, Jing F, Yang Y, Du L, Zheng G, Li P, Li C, Wang C. Exosomal long noncoding RNA CRNDE-h as a novel serum-based biomarker for diagnosis and prognosis of colorectal cancer. Oncotarget. 2016; 7:85551-85563. https://doi.org/10.18632/oncotarget.13465.

11. Qi P, Zhou XY, Du X. Circulating long non-coding RNAs in cancer: current status and future perspectives. Mol Cancer. 2016; 15:39.

12. Zhang Y, Xu YZ, Sun N, Liu JH, Chen FF, Guan XL, Li A, Wang F, Zhao QF, Wang HY, Song SS, Yu W, Zhao JN, et al. Long noncoding RNA expression profile in fibroblastlike synoviocytes from patients with rheumatoid arthritis. Arthritis Res Ther. 2016; 18:227.

13. Jiang H, Qin XJ, Li WP, Ma R, Wang T, Li ZQ. LncRNAs expression in adjuvant-induced arthritis rats reveals the potential role of LncRNAs contributing to rheumatoid arthritis pathogenesis. Gene. 2016; 593:131-142.

14. Chandra Gupta S, Nandan Tripathi Y. Potential of long non-coding RNAs in cancer patients: From biomarkers to therapeutic targets. Int J Cancer. 2016.

15. Sigdel KR, Cheng A, Wang Y, Duan L, Zhang Y. The Emerging Functions of Long Noncoding RNA in Immune Cells: Autoimmune Diseases. J Immunol Res. 2015; 2015:848790.

16. Chen D, Liu J, Zhao HY, Chen YP, Xiang Z, Jin X. Plasma long noncoding RNA expression profile identified by microarray in patients with Crohn's disease. World J Gastroenterol. 2016; 22:4716-4731.

17. Shi H, Cao N, Pu Y, Xie L, Zheng L, Yu C. Long non-coding RNA expression profile in minor salivary gland of primary Sjogren's syndrome. Arthritis Res Ther. 2016; 18:109.

18. Rosa A, Ballarino M. Long Noncoding RNA Regulation of Pluripotency. Stem Cells Int. 2016; 2016:1797692.
19. Wapinski O, Chang HY. Long noncoding RNAs and human disease. Trends Cell Biol. 2011; 21:354-361.

20. Sahu A, Singhal U, Chinnaiyan AM. Long noncoding RNAs in cancer: from function to translation. Trends Cancer. 2015; 1:93-109.

21. Kornfeld JW, Bruning JC. Regulation of metabolism by long, non-coding RNAs. Front Genet. 2014; 5:57.

22. Su DN, Wu SP, Chen HT, He JH. HOTAIR, a long noncoding RNA driver of malignancy whose expression is activated by FOXC1, negatively regulates miRNA-1 in hepatocellular carcinoma. Oncol Lett. 2016; 12:4061-4067.

23. Taucher V, Mangge H, Haybaeck J. Non-coding RNAs in pancreatic cancer: challenges and opportunities for clinical application. Cell Oncol (Dordr). 2016; 39:295-318.

24. Lee JT. Epigenetic regulation by long noncoding RNAs. Science. 2012; 338:1435-1439.

25. Zhou X, Han X, Wittfeldt A, Sun J, Liu C, Wang X, Gan LM, Cao H, Liang Z. Long non-coding RNA ANRIL regulates inflammatory responses as a novel component of NFkappaB pathway. RNA Biol. 2016; 13:98-108.

26. Ruan X. Long Non-Coding RNA Central of Glucose Homeostasis. J Cell Biochem. 2016; 117:1061-1065.

27. Aterido A, Palacio C, Marsal S, Avila G, Julia A. Novel insights into the regulatory architecture of CD4+ T cells in rheumatoid arthritis. PLoS One. 2014; 9:e100690.

28. Song J, Kim D, Han J, Kim Y, Lee M, Jin EJ. PBMC and exosome-derived Hotair is a critical regulator and potent marker for rheumatoid arthritis. Clin Exp Med. 2015; 15:121-126.

29. Bernstein EJ, Barr RG, Austin JH, Kawut SM, Raghu G, Sell JL, Hoffman EA, Newell JD Jr, Watts JR Jr, Nath PH, Sonavane SK, Sonavane SK, Bathon JM, et al. Rheumatoid arthritis-associated autoantibodies and subclinical interstitial lung disease: the Multi-Ethnic Study of Atherosclerosis. Thorax. 2016.

30. Xu L, Feng X, Tan W, Gu W, Guo D, Zhang M, Wang F. IL-29 enhances Toll-like receptor-mediated IL-6 and IL-8 production by the synovial fibroblasts from rheumatoid arthritis patients. Arthritis Res Ther. 2013; 15:R170.

31. Duffy L, O'Reilly SC. Toll-like receptors in the pathogenesis of autoimmune diseases: recent and emerging translational developments. Immunotargets Ther. 2016; 5:69-80.

32. Liu Y, Yin H, Zhao M, Lu Q. TLR2 and TLR4 in autoimmune diseases: a comprehensive review. Clin Rev Allergy Immunol. 2014; 47:136-147.

33. Hsu YL, Wang MY, Ho LJ, Lai JH. Dengue virus infection induces interferon-lambda1 to facilitate cell migration. Sci Rep. 2016; 6:24530.

34. Lee HC, Narayanan S, Park SJ, Seong SY, Hahn YS. Transcriptional regulation of IFN-lambda genes in hepatitis $\mathrm{C}$ virus-infected hepatocytes via IRF-3.IRF-7.NF-kappaB complex. J Biol Chem. 2014; 289:5310-5319. 